\title{
Comparative Analyses of Pharmaceuticals or Food Supplements Containing Chondroitin Sulfate: Are Their Bioactivities Equivalent?
}

\author{
Antonietta Stellavato - Odile Francesca Restaino - Valentina Vassallo • \\ Rosario Finamore - Carlo Ruosi • Elisabetta Cassese · Mario De Rosa • \\ Chiara Schiraldi iD
}

Received: July 2, 2019 / Published online: September 7, 2019

(C) The Author(s) 2019

\begin{abstract}
Introduction: Oral supplementation of chondroitin sulfate (CS) and glucosamine $(\mathrm{GlcN})$, symptomatic slow-acting molecules, is recommended by European Society for Clinical and Economic Aspects of Osteoporosis and Osteoarthritis and Musculoskeletal Diseases (ESCEO) and other European Union (EU) guidelines for the restoration of the articular cartilage surface in patients affected by osteoarthritis (OA). They are commercialized as pharmaceutical grade products and as food supplements in combination with plant extracts hyaluronic acid, methylsulfonylmethane, and
\end{abstract}

Enhanced Digital Features To view enhanced digital features for this article go to https://doi.org/10.6084/ m9.figshare.9448109.

A. Stellavato $(\varangle) \cdot$ O. F. Restaino · V. Vassallo .

R. Finamore · E. Cassese · C. Schiraldi ( $\square)$

Department of Experimental Medicine, Section of

Biotechnology and Molecular Biology, University of Campania "Luigi Vanvitelli", Via De Crecchio 7, 80138 Naples, Italy

e-mail: antonietta.stellavato@unicampania.it

C. Schiraldi

e-mail: chiara.schiraldi@unicampania.it

C. Ruosi

Department of Public Health, School of Medicine and Surgery "Federico II" of Naples, A.O.U. Federico

II of Naples, Via S. Pansini, 80131 Naples, Italy

M. De Rosa

Bioteknet, Via De Crecchio 7, 80138 Naples, Italy other components. Food supplements do not need to undergo the strict regulatory controls of pharmaceutical grade products; thus, composition and contaminants that could be present may not be evidenced before commercialization and these uncertainties may give rise to concerns about the bioactivity of these formulations.

Methods: In this paper 10 different food supplements (FS) from diverse European countries were analyzed in comparison with two pharmaceutical grade products $(\mathrm{Ph})$ using updated analytical approaches and biochemical cellbased assays. The purity, the titer, and the origin of CS in Ph and FS samples were initially assessed in order to successively compare the biological function. Both food supplements and pharmaceutical formulations were tested in vitro, using the same final CS concentration, on primary chondrocytes and synoviocytes in terms of (i) cell viability, (ii) activation of the

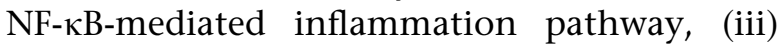
cartilage oligomeric matrix protein (COMP-2), IL-6, and IL-8 production.

Results: All the FS presented a certain insoluble fraction; the CS and the GlcN contents were lower than the declared ones in $9 / 10$ and $8 / 10$ samples, respectively. All FS contained keratan sulfate (KS) at up to $50 \%$ of the total glycosaminoglycan amount declared on the label. Primary cells treated with the samples diluted to present the same CS concentration in the medium showed cytotoxicity in 7/10 FS while 
$\mathrm{Ph}$ preserved viability and reduced NF- $\kappa \mathrm{B}$, COMP-2, and secreted inflammatory cytokines. Conclusion: Among all samples tested, the pharmaceutical grade products demonstrated effective modulation of biomarkers counteracting the inflammation status and improving viability and the physiological condition of OA human primary chondrocyte and synoviocyte cells. In contrast to that, most FS were cytotoxic at the tested concentrations, and only $3 / 10$ of them showed similarities to $\mathrm{Ph}$ sample behavior in vitro. Funding: This work was partially supported by PON01_1226 NUTRAFAST, MIUR Ministero dell'Università e della Ricerca Scientifica. Bioteknet financed two short-term grants for graduate technicians. The journal's Rapid Service and Open Access fees were funded by IBSA CH.

Keywords: Chondroitin sulfate; Food supplements; Glucosamine; HPAE-PAD; Human primary chondrocytes; Human primary synoviocytes; Keratan sulfate; NF- $\mathrm{KB}$ mediated inflammation pathway; $\mathrm{OA}$ in vitro model; Rheumatology

\section{INTRODUCTION}

Osteoarthritis (OA) is essentially a debilitating musculoskeletal disease with a high public health impact. Also known as degenerative joint disease, OA is characterized by a progressive loss of articular cartilage in synovial joints [1]. The major consequence is several functional limitations such as joint stiffness, and it is associated with inflammation of the synovial membrane. In fact, the importance of synovitis in OA progression has gradually been recognized [2]. It is estimated that this pathology affects 40 million Europeans and 30 million Americans over the age of 50 and it is expected to increase by up to $35 \%$ with the aging of the population [3]. The pathogenesis of OA starts with a mechanical overloading of the articular joints, cartilage degradation, joint space narrowing, and loss of cartilage and synovial fluids, and thus bone degradation [3, 4]. Specific mediators produced during OA can directly act on cartilage and on multiple signaling pathways activating other key inflammatory molecules [4]. In fact, the nuclear factor kappa-light-chain-enhancer of activated $\mathrm{B}$ cell (NF- $\kappa \mathrm{B})$ pathway is associated with OA pathophysiology $[4,5]$, prompting the expression of the inflammatory mediators (Fig. 1). These include interleukin- 1 beta (IL-1 $\beta$ ), tumor necrosis factor alpha (TNF- $\alpha$ ), interleukin-6 (IL-6), interleukin-8 (IL-8), cyclooxygenase-2 (COX2), chemokines, and colony-stimulating factors $[6,7]$.

Current OA pharmacological therapy is directed at the prevention of pain and the improvement of function. Standard treatments
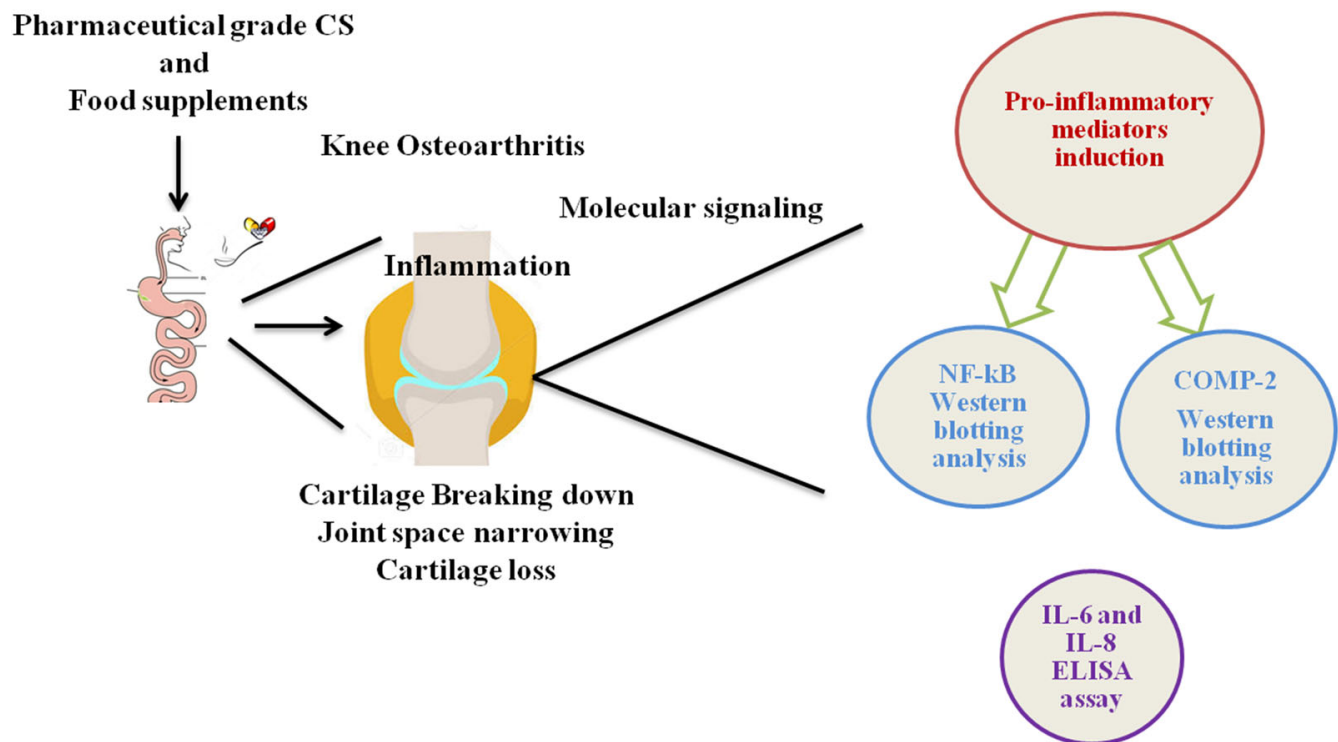

Fig. 1 Schematic view of the experimental setup 
Table 1 Chondroitin sulfate and glucosamine origin, structure, and suggested posology

\begin{tabular}{|c|c|c|}
\hline & Chondroitin sulfate (CS) & Glucosamine (GlcN) \\
\hline Structure & $\begin{array}{l}\text { 4)- } \beta \text {-GlcA- }(1 \rightarrow 3)-\beta-G a l N A c-(1 \rightarrow \\
\text { Terrestrial origin } \mathrm{CS} M_{\mathrm{w}}=14-26 \mathrm{kDa} ; 4 \mathrm{~S} / \\
6 \mathrm{~S}=1.3-5.0 \\
\text { Marine origin } \mathrm{CS} M_{\mathrm{w}}=30-80 \mathrm{kDa} ; 4 \mathrm{~S} / \\
6 \mathrm{~S}=0.3-1.8 \\
{[17,18]}\end{array}$ & $\mathrm{GlcN} M_{\mathrm{w}}=215 \mathrm{Da}$ \\
\hline Biological role & $\begin{array}{l}\text { The CS is a glycosaminoglycan (GAG) found in the } \\
\text { extracellular matrix of the animal cartilaginous } \\
\text { tissues }\end{array}$ & $\begin{array}{l}\text { In the human body the GlcN is produced as } \\
\text { precursor of glycosylated lipids and proteins, } \\
\text { GAGs, and proteoglycans }\end{array}$ \\
\hline Origin & $\begin{array}{l}\text { CS is manufactured by extraction and purification } \\
\text { from the animal cartilaginous tissues of shark fins, } \\
\text { bovine and chicken trachea, or pig muzzle }\end{array}$ & $\begin{array}{l}\text { GlcN is manufactured from chitin of crustacean } \\
\text { shells by both alkaline or acid hydrolysis or by de } \\
\text { novo chemical synthesis, and it is generally sold as } \\
\text { glucosamine hydrochloride or glucosamine sulfate } \\
\text { salts [9] }\end{array}$ \\
\hline $\begin{array}{c}\text { Recommended } \\
\text { daily dosage }\end{array}$ & $800-1200 \mathrm{mg}$ & $1250-1500 \mathrm{mg}$ \\
\hline
\end{tabular}

mainly include a combination of pharmacological and non-pharmacological methods, like physical exercises, physiotherapy, weight loss, etc. The first choice has been pharmacological therapy with analgesics or non-steroidal antiinflammatory drugs (NSAIDs) essentially directed towards the prevention and the control of pain and the improvement of motility function in patients affected by OA [8]. However, numerous studies reported that the prolonged use of NSAIDs could cause adverse effects in the gastrointestinal and cardiovascular systems. For this reason-and bearing in mind that the patient treatment is prolonged for years-the need has developed for new therapeutic targets for OA treatment that could relieve pain and potentially reduce the damage. As an alternative to NSAIDs, chondroitin sulfate (CS) and glucosamine (GlcN), which are natural extractive compounds, have been adopted $[9,10]$ since the 1990s and recently recommended in the European Society for Clinical and Economic Aspects of Osteoporosis and Osteoarthritis and Musculoskeletal Diseases (ESCEO) algorithm as preferential treatments at the initial stage of OA. Several clinical trials have reported the efficacy of CS and GlcN in delaying joint damage, in pain reduction, and in improving articular mobility in patients with OA. Unlike NSAIDs, prescription $\mathrm{CS}$ and/or GlcN drugs are able to delay the progression of the pathology or eventually reverse morphological changes in joint structure besides working as Symptomatic Slow-Acting Drugs for OA (SySADOAs) and Disease-Modifying OsteoArthritis Drugs (DMOADs) [11]. The recommended daily dosages for CS are between 800 and $1200 \mathrm{mg}$ while the daily dosages for GlcN range from 1250 to $1500 \mathrm{mg}$ (Table 1). At present they are recommended by the European League Against Rheumatism (EULAR) and ESCEO as 
SySADOAs for the treatment of knee, hand, or hip osteoarthritis, providing pain relief and increasing joint mobility $[12,13]$. Numerous food supplements containing CS and/or GlcN are on the market and they are becoming an increasingly popular alternative approach to prescription drugs especially among those patients who prefer non-pharmacological treatment options. However, there is still an unsolved problem related to the quantitative and qualitative control of CS and GlcN contained in food supplements and nutraceuticals [14-16].

In the absence of any mandatory analytical controls from European Medicines Agency (Eur. Pharmacopeia) and of other specific national/ international regulatory rules or of specific industrial guidelines, each manufacturer has been able to establish its own analytical procedures for the determination of chondroitin sulfate and glucosamine identity, content, and purity in food supplements [19-22]. Recent papers have also demonstrated that keratan sulfate (KS), which is another component of animal articular cartilaginous tissue, may be extracted along with the CS, thus contaminating it [23]. Because of the structural similarities, and the superimposable size of CS and KS biopolymers, the selective removal of this macromolecule is challenging [24-26]. It is of great scientific interest for the assessment of analytical strategies to fully characterize food supplement purity, with the specific target of finding a correlation between structural characteristic and biological activity using in vitro models [27]. In fact, robust data, generated in well-assessed models, may better permit the critical analysis of the clinical scientific literature to ascertain the validity of the guidelines on a strong scientific basis. Often the SySADOAs have been regarded as "questionable" products with controversial clinical outcomes. However, it was pointed out that the variability in the beneficial effects in patients with OA is strongly reduced, ascertaining effectiveness of CS-based treatments, when referring to pharmaceutical grade products [28].

In this study, $10 \mathrm{FS}$ commercialized in eight different European countries were extensively analyzed and compared to pharmaceutical CS with a specifically assessed experimental design on three human primary cell models.

\section{METHODS}

This article is not based on in vivo studies, on either animals or humans. However, the cells used were obtained by digestion of joint/cartilage tissues harvested from surgical procedures on human participants. The pieces would have been wasted and, as part of a collaboration scheme, these were used instead to set up in vitro models used in this experimental research project. The protocol was approved by the ethical committee of the University (AOU-SUN) (protocol registration No. $0003711 / 2015$ of the $16 / 05 /$ 2015; study title "Glicosamminoglicani e/o derivati biotecnologici utili alla rigenerazione tessutale"). Informed consent was obtained from all participants involved in this study.

\section{Reagents}

Sodium nitrate, sodium acetate, $\mathrm{N}$-acetylgalactosamine, glucosamine, galactose, and glucuronic acid were from Sigma-Aldrich (USA). The $\mathrm{NaOH}$ solution was from J.T. Baker (Netherlands). The hydrochloric acid was from Carlo Erba (Italy). The medium and the enzymes used in the biological experiments for cell cultures were from Gibco, Invitrogen (USA), unless otherwise specified. The 10 food supplements (FS), containing both CS and GlcN, and the two pharmaceuticals $(\mathrm{Ph})$, containing only CS, that were from different European countries and companies, were either purchased or obtained as test/gift samples. Cell culture reagents and enzymes used in the biological experiments were from Gibco, Invitrogen (USA), unless otherwise specified.

\section{Analytical Evaluation: Structural Characterization and Composition}

A single dose of FS (one capsule or tablet) was dissolved in $20 \mathrm{ml}$ of MilliQ water, in triplicate, placed and stirred at $300 \mathrm{rpm}$, at room temperature for $6 \mathrm{~h}$ and then left overnight to 
sediment. The next day, the samples were photographed and then centrifuged at 6500 rpm for 30 min (Avanti J20-XP, Beckman Coulter, USA) to separate the supernatants from the insoluble fractions which were then dried under vacuum at $40^{\circ} \mathrm{C}$ for $24 \mathrm{~h}$ (Binder $\mathrm{GmbH}$, Germany) and weighed. The insoluble solid weight values were calculated as a ratio of the weight of the insoluble portion of the sample to the total dry weight, as a percentage. The supernatants were filtered on $0.22-\mu \mathrm{m}$ filters (Millipore, France) and the protein content was determined according to the Bradford method [29], following a protocol previously described [23].

\section{Chromatographic Profiles by Strong Anion Exchange (SAX) Chromatography}

Each sample (one capsule or tablet) was dissolved in $20 \mathrm{ml}$ of buffer A $(20 \mathrm{mM}$ sodium acetate, $0.5 \mathrm{M}$ sodium chloride, $\mathrm{pH} 7.4$ ), centrifuged at $6500 \mathrm{rpm}$ for $30 \mathrm{~min}$ (Avanti J20-XP, Beckman Coulter, USA), and the supernatant was separated from the insoluble portion. For each sample, $2 \mathrm{ml}$ of the supernatant were microfiltered on $0.22-\mu \mathrm{m}$ filters (Millipore, France) and loaded on an anion-exchange column (HiPrep Q Sepharose 16/10 HP, GE Healthcare, Milan, Italy) connected to an ÄKTA purifier system (GE Healthcare, Milan, Italy), previously equilibrated with buffer A. Samples were then eluted with buffer B $(20 \mathrm{mM}$ sodium acetate, $3.0 \mathrm{M}$ sodium chloride, $\mathrm{pH}$ 7.4) applying a linear gradient. The chromatograms were obtained by recording a signal at $215 \mathrm{~nm}$ and the peak areas were used to calculate the representativeness of each peak in each sample as the percentage ratio of the area of the single peak divided by the sum of the areas of all the peaks present in the chromatograms [23]. Pie charts were created by using these instances of representativeness.

\section{HPAE-PAD Analyses}

Determination of the CS and GlcN contents of the sample supernatants and of their eventual KS contamination was performed by high- performance anion-exchange chromatography (HPAE-PAD) analysis on the basis of the monosaccharide composition, according to a previously reported method [23]. The CS content was determined after a hydrolytic procedure on the supernatants as previously described [23]; the GlcN monosaccharide content was determined after ultrafiltering the sample supernatants on $3-\mathrm{kDa}$ membranes, according to a previously reported protocol $[23,26]$ and analyzing the permeate fractions. The retentate fraction that contained the CS and the eventual KS was hydrolyzed and analyzed as well. Quantitative analyses were based on determination of the GalNAc concentration for the CS content, of the free GlcN concentration for the GlcN monosaccharide content, or of the bound GlcN concentration for the KS contamination.

\section{Biological Activity}

Bioactivity of food supplements in comparison to CS pharmaceutical-based products was assayed using three different cellular models: (I) chondrocytes isolated from nasoseptal cartilage of healthy human patients and exposed to IL-1 $\beta(10 \mathrm{ng} / \mathrm{mL})$ in order to mimic in vitro inflammatory conditions [30, 31]; (II) articular chondrocytes; and (III) synoviocytes that were isolated from knee cartilage and synovial fluid of patients affected by OA who underwent a joint replacement surgical procedure at the Orthopedics and Traumatology Department of the Federico II University of Naples, as previously reported [32].

\section{Isolation and Culture of Cartilage Chondrocytes and Knee Joint Synoviocytes}

\section{1st Model}

Nasoseptal cartilage was obtained from surgical procedures on functional respiratory airways. Patients did not present cartilage alteration (e.g., OA, etc.). The protocol assessed in our lab was previously reported [30]. Briefly, minced cartilage was digested using an enzymatic solution (collagenase type I at $3 \mathrm{mg} / \mathrm{ml}$ and dispase 
at $4 \mathrm{mg} / \mathrm{ml}$ ), diluted in PBS, and gentamicin $0.2 \mathrm{mg} / \mathrm{ml}$ (Hospira, IL, USA) at a temperature of $37^{\circ} \mathrm{C}$ on a shaking plate overnight. The cells were separated from undigested pieces through a sterile filter $(70 \mu \mathrm{m}, \mathrm{BD}$ Biosciences Bedford, USA) and the cellular suspension was centrifuged at $1500 \mathrm{rpm}$ for $7 \mathrm{~min}$ (Eppendorf Centrifuge, Milan, Italy). Cells were then washed with PBS, re-centrifuged, and re-suspended in DMEM supplemented with fetal bovine serum (FBS, 10\%). Fluorescence-activated cell sorting (FACS) analyses were performed to characterize the cell phenotype [30].

\section{2nd and 3rd Models}

In particular, two different kinds of cartilage from the same knee were obtained: a piece of intact cartilaginous tissue that represents the healthy part and was considered the control (healthy control), while the other samples were harvested from damaged cartilage tissue of the same knee (pathological control). Serum (FBS) $(10 \% \mathrm{v} / \mathrm{v})$, penicillin-streptomycin $(1 \% \mathrm{v} / \mathrm{v})$, and amphotericin B (1\% v/v) (Lonza, Basel, Switzerland).

Digestion was accomplished following the same protocol describe above, and previously published [32]. The cells were seeded in a $35-\mathrm{mm}$ tissue culture well and maintained at $37^{\circ} \mathrm{C}$ in a humidified atmosphere with $5 \% \mathrm{v} / \mathrm{v}$ $\mathrm{CO}_{2}$. Synoviocyte cells were isolated from the synovial fluid. Specifically, synovial fluid samples were centrifuged, the supernatant was removed, and the pellet was washed with PBS. The derived cells were seeded, after characterization using flow cytometry according to the previously reported protocol [32, 33].

\section{Cell Viability Assay}

All three models described above were used for a viability assay in the presence of the $\mathrm{FS}$ and $\mathrm{Ph}$ products. Trypan blue exclusion assay was used to discriminate viable cells from non-viable ones; $2 \times 10^{4}$ cells were seeded in 12-well plates (BD Falcon, USA) to obtain a consistent monolayer. In particular, after $48 \mathrm{~h}$ of incubation the culture medium was removed and then replaced by either fresh medium alone (control), or medium containing the food supplements or the pharmaceutical grade chondroitin sulfate samples. All the samples (FS1, FS2, FS3, FS4, FS5, FS6, FS7, FS8, FS9, FS10, Ph1, and Ph2) were tested at the same CS concentration $(4 \mathrm{mg} / \mathrm{mL})$. After $48 \mathrm{~h}$, cells were harvested with trypsin, centrifuged, and stained with trypan blue (Sigma Aldrich, Milan, Italy) following the manufacturer's instructions. Viable cells (not colored) and dead cells (blue) were counted by using a Bürker chamber from two different independent observers. Cell viability (\%) was expressed as the number of viable cells/the number of total cells $\times 100$.

\section{Quantification of IL-6 and IL-8 Production Using ELISA}

Cell supernatants were collected after $48 \mathrm{~h}$ of treatment, centrifuged (3000 rpm for $10 \mathrm{~min}$ at $4{ }^{\circ} \mathrm{C}$ ), and analyzed to quantify IL-6 and IL-8 production. The cytokines were assayed using an ELISA (Boster Biological Technology Pleasanton, USA). Each experiment was performed in triplicate and cytokine amounts were determined using a microplate reader (Biorad laboratories, Milan, Italy). The analytic concentrations were calculated using a standard curve according to the manufacturer's instructions and as previously reported [33].

\section{Protein Extraction and Western Blot Analyses}

The cells' intracellular total protein content was extracted in the radioimmunoprecipitation assay (RIPA buffer) $(1 \times)$ (Cell Signaling Technology, USA). Protein concentration was determined using the Bradford method (Biorad Laboratories, Milan, Italy) and $5 \mu \mathrm{g}$ of proteins was separated by means of sodium dodecyl sulfate polyacrylamide gel electrophoresis, transferred onto nitrocellulose membranes (Millipore, Bedford, MA), and blocked with 5\% skimmed milk in Tris-buffered saline with Tween 20 (TBST). The membranes were incubated with primary antibodies to detect COMP2 (Abcam, Cambridge, UK) and NF-кB (Santa Cruz Biotechnology, CA, USA) both used at 1:500 dilutions and incubated overnight at $4{ }^{\circ} \mathrm{C}$. 
After washing with TBST, immunolabeling bands were detected by the chemiluminescence detection system using corresponding horseradish peroxidase-conjugated secondary antibodies (Santa Cruz Biotechnology, CAUSA), diluted $1: 10,000$ for $1 \mathrm{~h}$ at room temperature and reacted with an ECL system (Merck Millipore, Darmstadt Germany). Protein levels were normalized with respect to the signal obtained with an anti-actin antibody 1:500 dilution (Santa Cruz Biotechnology, CA, USA). The semiquantitative analyses of protein levels were carried out using the Gel Doc 2000 UV System (Biorad laboratories, Milan, Italy) according to the manufacturer's protocols and previously reported [32, 33].

\section{RESULTS}

\section{Insoluble Solid Determination and Protein Content Analyses}

As can be seen from Table 2, Ph and FS samples look very different in terms of color, transparency, and sediment. In fact, FS solubility is lower than that of analyzed Ph products. The latter showed transparent rather than colored solutions while FS suspensions were yellow-orange colored and opaque (photo in Table 2). The 10 FS samples were analyzed to evaluate the protein content. In sample FS1 the quantity of proteins found is $3.9 \%$ of the declared value. The presence of proteins was identified in three of the five FS samples (Table 2) even if not clearly stated on the labels.

\section{Chromatographic Profiles by SAX Chromatography}

The chromatographic profiles of the FS samples obtained by SAX chromatography were used to create a pie chart in which the percentage ratio of the area of every single peak divided by the sum of all the peak areas was reported (Fig. 2). Generally three families of peaks were visible in the chromatograms: the unretained (UR) peak containing the species not bound to the stationary phase, the chondroitin sulfate (CS) peak, and the other component peaks (OC). A representative chromatogram is shown in Fig. 2. As visible in the graphs (Fig. 2), in Ph1 and Ph2 samples the CS peak had a percentage area higher than $80 \%$ while among the FS samples only FS5 showed a CS peak higher than $60 \%$. The others showed lower CS percentages with the lowest value of $3.1 \%$ being in the case of FS7.

\section{CS, GlcN Content, and KS Contamination by HPAE-PAD Analyses}

The CS and GlcN contents in the food supplements and the eventual residual KS contamination were determined by HPAE-PAD analyses and data were compared with the pharmaceutical grade samples. The CS content in the food supplements was lower than the declared content in nine samples out of 10 , with differences ranging from 5.0 to $145.6 \mathrm{mg}$ (Table 2); the GlcN content was lower the declared contents in eight samples out of 10 with differences ranging from 3.0 to $130.4 \mathrm{mg}$ (Table 2). All the samples were contaminated with $\mathrm{KS}$ in the range from $11.7 \%$ to $47.9 \%$ of the total amount of GAGs present (Table 2). The pharmaceutical grade samples showed a CS content consistent with the declared content and a KS contamination of $2.0 \%$ maximum (Table 2 ).

\section{Biological Activity}

\section{First Model: Nasal Chondrocytes}

Cell Viability Food supplements were assayed on IL-1 $\beta$-challenged nasal chondrocytes $[29,32]$. Cell viability was slightly reduced in the presence of IL-1 $\beta$ (65\%) with respect to control. Ph1 and Ph2 particularly improved viability and among the FS tested only three (FS2, FS3, and FS6) supported growth. Specifically, the percentage of viable cells found for all three was in the range $60-70 \%$, with respect to untreated cells (CTR) (Fig. 3a).

Cytokine Quantification: IL-6 and IL-8 Protein Expression in Cell Supernatants Results showed that, as expected, IL-1 $\beta$ treatment increased both IL-6 and IL-8 level by about 2.5fold and 1.6-fold, respectively, with respect to 


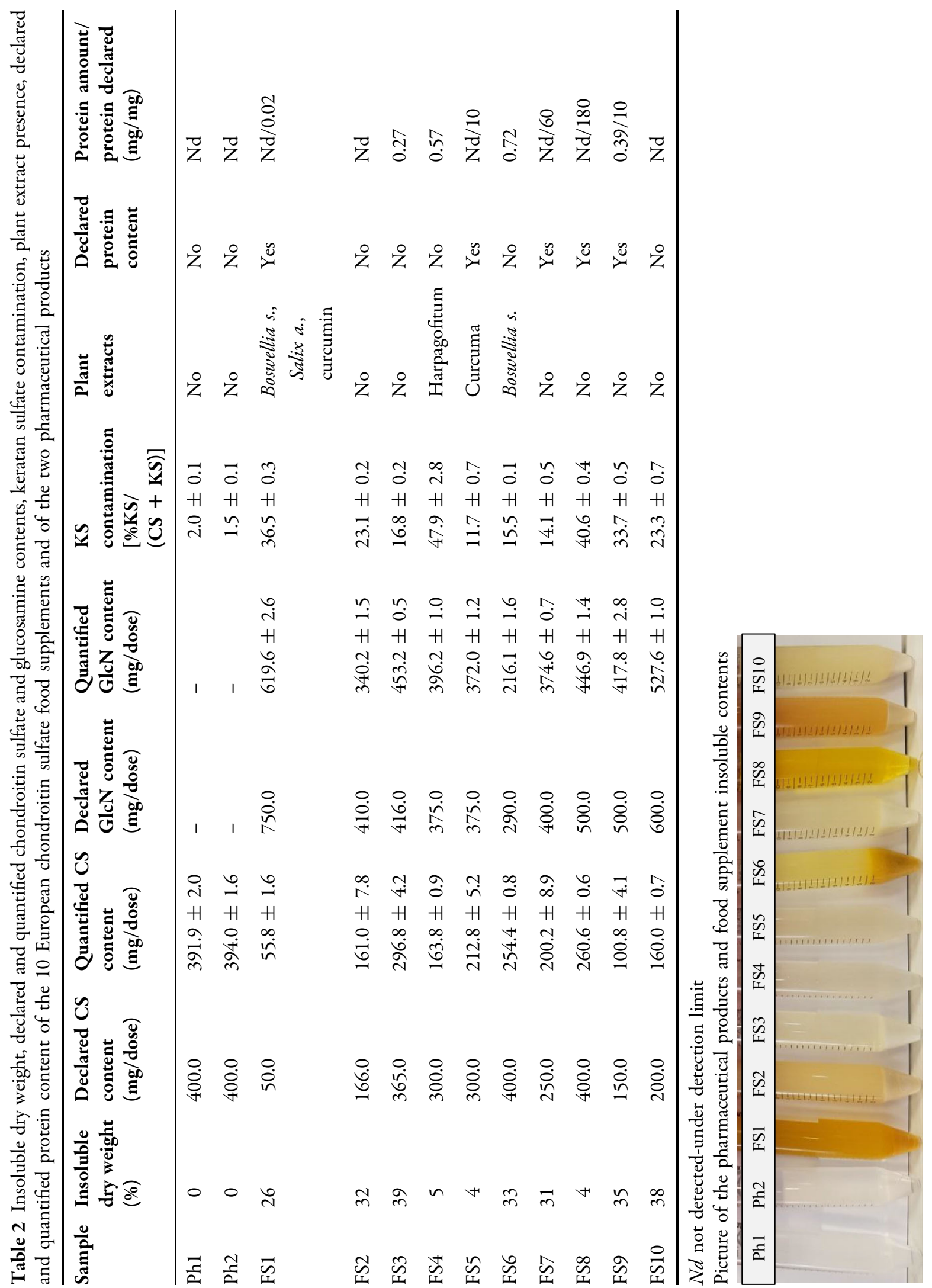




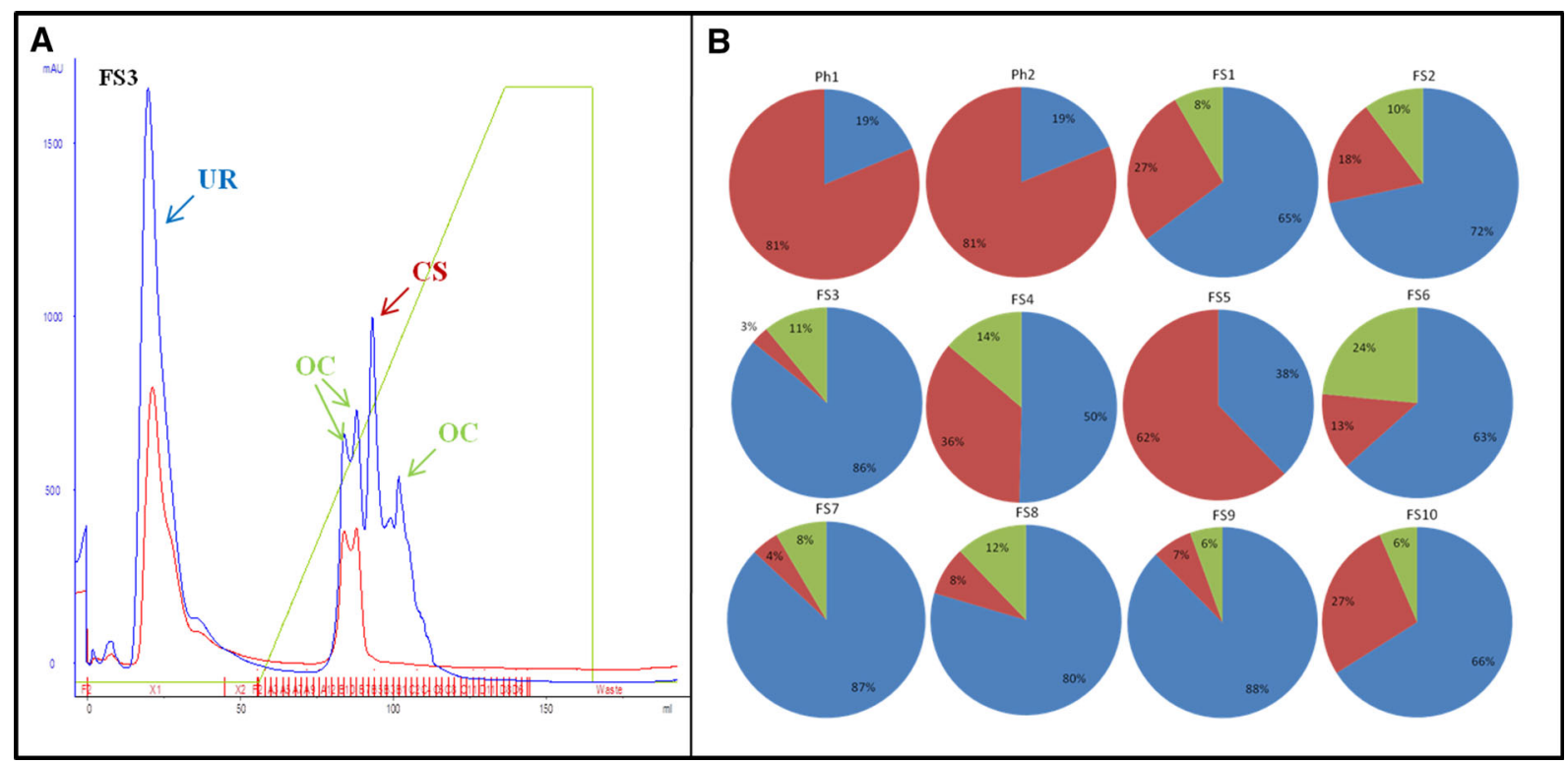

Fig. 2 a FS4 strong anion exchange chromatography profiles: in blue, absorbance at $215 \mathrm{~nm}$; in red, at $280 \mathrm{~nm}$. b Graphs obtained from the ratios of the peak areas at $215 \mathrm{~nm}$ : UR unretained peak, OC other component peak, CS chondroitin sulfate peak. UR peak may

CTR. Beside, Ph1 and Ph2 significantly reduced IL-6 expression by 5-fold and 4.5-fold, respectively, with respect to IL-1 $\beta$ challenged cells. Among FS samples only the three that proved not to be cytotoxic at the concentrations tested showed their efficacy as anti-inflammatory agents. In fact, FS2, FS3, and FS6 reduced IL-6 production with respect to IL-1 $\beta$ treatments (Fig. 3b). For IL-8, both pharmaceutical products, Ph1 and Ph2, reduced production by 2.4 fold and 9.8-fold, respectively. Among food supplement samples, FS6 was not able to reduce IL-8 expression, whereas FS2 and FS3 reduced IL-8 by about fivefold with respect to the negative control (Fig. 3b).

\section{Second Model: Articular chondrocytes}

Cell Viability Cell viability was lower for primary chondrocytes (Fig. 4a) isolated from very damaged joint cartilage of patients with OA (pCTR) with respect to the ones isolated from less damaged parts (considered as healthy). Ph1 and $\mathrm{Ph} 2$ improved the condition, with $65-75 \%$ of counted viable cells. Among the FS tested, only three (FS2, FS3, and FS6) proved not contain non-sulfated CS, KS, and/or other components; OC peaks may contain glucosamine, proteoglycans, and keratin sulfate (in the last peak); CS peak contains chondroitin sulfate as reported by [23]

detrimental to cell growth, providing at least $65 \%$ of viable cells as reported in Fig. $4 \mathrm{~b}$.

Cytokine Quantification: IL-6 and IL-8 Protein Expression in Cell Supernatants Results of ELISAs (Fig. 4c) showed that IL-6 of pCTR was increased with respect to the healthy one as expected. Ph1 and Ph2 both reduced this marker by about twofold. Among the three FS selected, FS2 does not show any effect but FS3 prompted a 1.7 -fold reduction with respect to pCTR while FS6 reduces IL-6 by 1.2-fold. Concerning IL-8 expression, data showed that only $\mathrm{Ph} 1$ and $\mathrm{Ph} 2$ reduced this marker level with respect to pCTR by about 2.5 -fold and 1.5 -fold, respectively. In contrast, the food supplements showed a negative modulation of this biomarker.

COMP-2 and NF-кB Protein Expression: Western Blotting Experiments The cells obtained from damaged cartilage (pCTR) expressed higher levels of the pro-inflammatory protein NF- $\kappa B$ and COMP-2 than hCTRL, as evidenced by western blotting analyses (Fig. $4 \mathrm{~d}$ ). After $48 \mathrm{~h}$ 


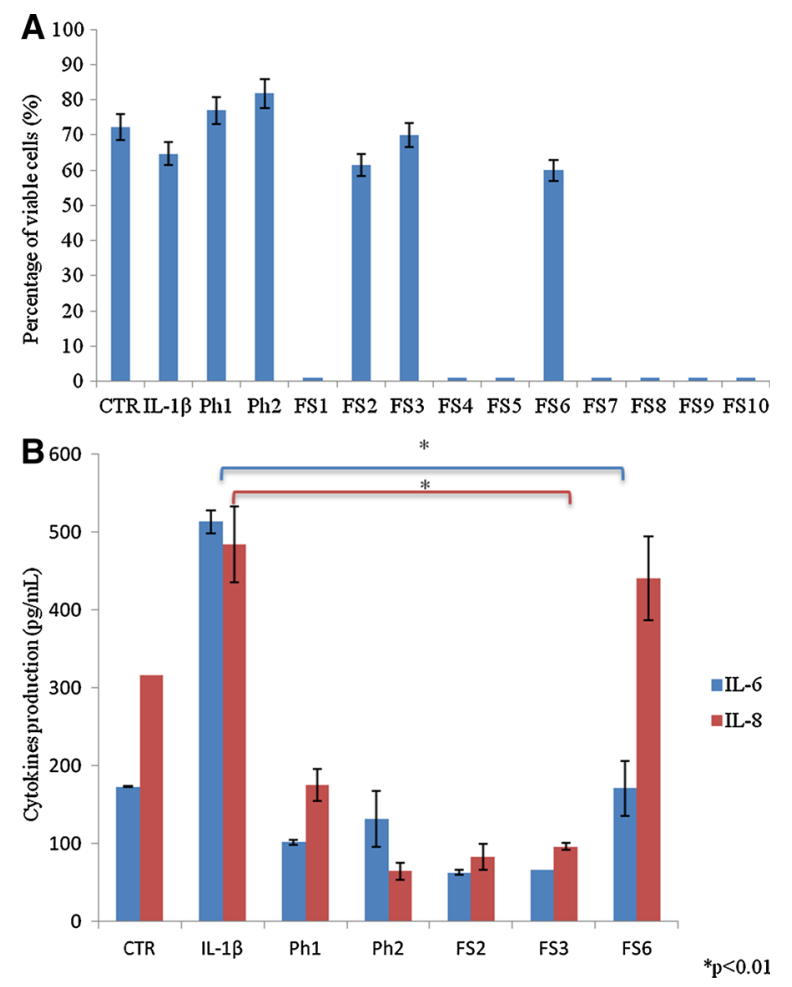

Fig. 3 a Nasal chondrocyte cell viability using trypan blue staining. b IL-6 and IL-8 cytokine quantification using ELISA. $T$ test analyses $\left({ }^{*} p<0.01\right)$ were performed to compare the significance of both pharmaceutical grade chondroitin sulfate samples and food supplements on IL-6 and IL- 8 production by nasal chondrocytes with respect to IL-1 $\beta$-treated cells

of treatment both with pharmaceutical products (Ph1 and Ph2) and food supplements, NF$\kappa \mathrm{B}$ and COMP-2 protein expression decreased. In particular, Ph1 and $\mathrm{Ph} 2$ showed a 1.3-fold and 1.5-fold reduction of NF- $\mathrm{KB}$ expression in comparison to pCTR, respectively. However, among the three FS that were not cytotoxic, FS3 was also able to reduce NF- $\mathrm{BB}$ expression by about 1.6-fold. Furthermore, as shown in Fig. 4d, Ph1 and Ph2 also slightly reduced the expression of COMP-2 by about 1.3-fold and 1.2-fold, respectively, with respect to pCTR. Among FS, FS6 reduced COMP-2 expression by 1.5-fold with respect to $\mathrm{p}$-CTR.

\section{Third Model: Synoviocytes}

Cell Viability The synoviocyte cell (Fig. 5a) based model does not have a healthy control
Fig. 4 Biological activity on articular chondrocytes. a Cell picture panel in the presence of food supplements and pharmaceutical grade products versus healthy and pathological CTR. b Cell viability using trypan blue staining. c IL-6 and IL-8 cytokine quantification through ELISA. d Western blotting analyses of NF- $\kappa \mathrm{B}$ and COMP-2 versus actin housekeeping protein normalization. $T$ test analyses $\left({ }^{*} p<0.01\right)$ were performed to compare the significance of both pharmaceutical grade CS samples and food supplements on IL- 6 and IL-8 production, on NF- $\mathrm{KB}$ and COMP-2 protein expression by articular chondrocytes with respect to pathological non-treated cells

since the cells are isolated from a population that is consistent with the pathological OA condition (membrane and fluid in the OA-affected joint). Cell viability (Fig. 5b) is about $65 \pm 7 \%$ after $48 \mathrm{~h}$ for the pCTR, as expected. Ph1 and Ph2 treatments improved viability by up to $85 \%$. Among FS tested, the cell viability percentage for FS5 is about 20\%, while for FS6 it is about $65 \%$. Results for FS2 and FS3 were better, with percentage of viable cells of about $80 \pm 10 \%$ for both.

\section{Cytokine Quantification: IL-6 and IL-8 Protein} Expression in Cell Supernatants In synoviocyte cells, IL-6 production (Fig. 5c) is significantly reduced in the presence of $\mathrm{Ph} 2$ by about 35 -fold with respect to pCTR. However, Ph1 is also able to counteract cytokine production, specifically by 2.2 -fold with respect to pCTR. Concerning food supplements, only the samples that reduce cell viability were considered for IL-6 quantification. In particular, FS2 reduced IL- 6 production by about 1.6 -fold with respect to pCTR; FS6 reduced IL-6 production by 2.2-fold, and FS3 reduced IL-6 production by about 30-fold.

Concerning IL-8, Ph2 proved the most effective but all the FS-treated samples afforded IL-8 concentrations not significantly modulated with respect to pathological control.

COMP-2 and NF-kb Protein Expression: Western Blotting Experiments We evaluated the modulation of the same biomarkers for the third implemented model. In this context the obtained cells were regarded as being subject to an ongoing inflammatory process. After $48 \mathrm{~h}$ of 


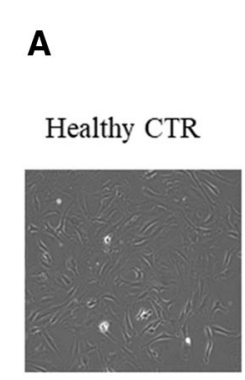

$\mathrm{Ph} 1$

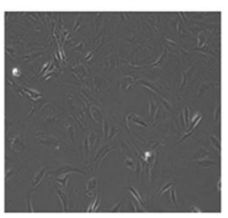

FS1

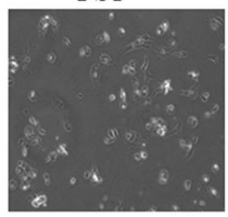

FS3

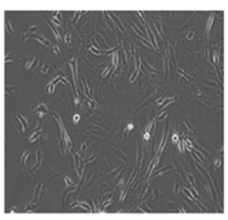

FS5

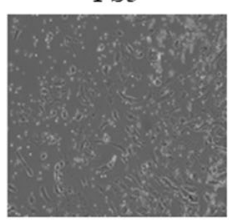

FS7

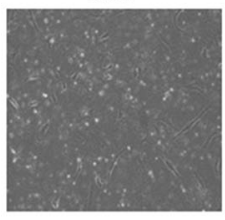

FS9

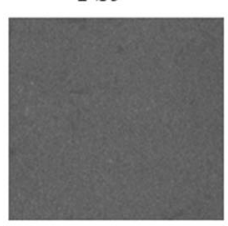

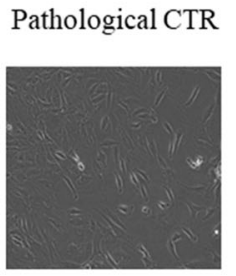

$\mathrm{Ph} 2$

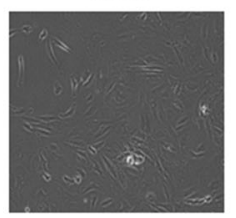

FS2

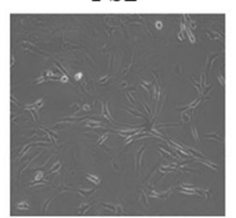

FS4

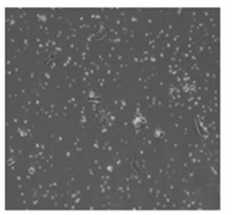

FS6

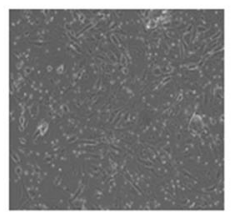

FS8

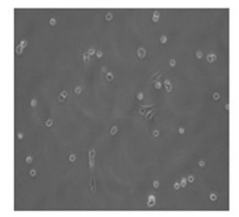

FS10

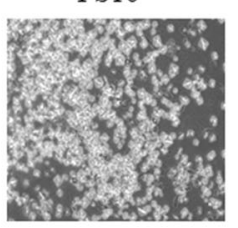

B

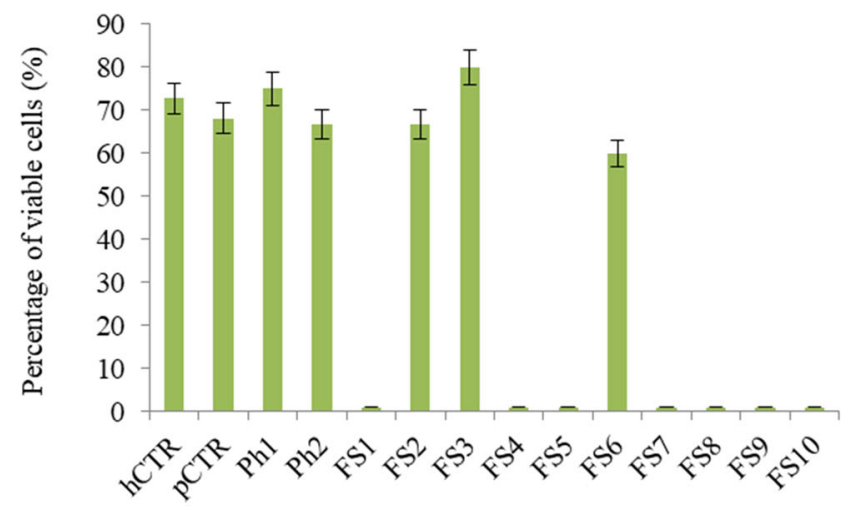

C
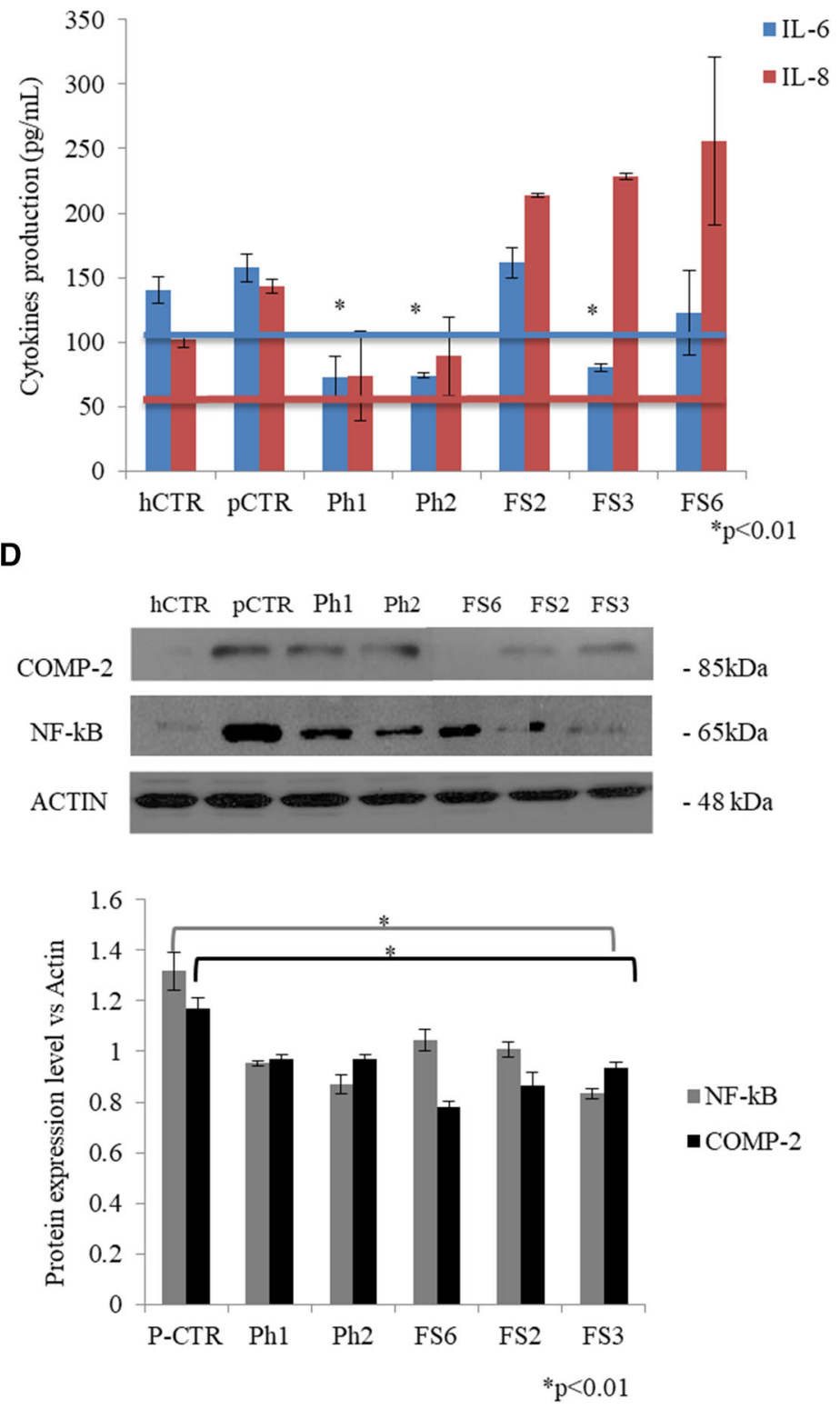
A

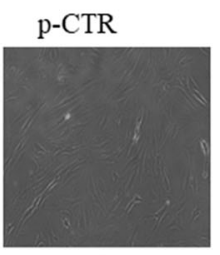

$\mathrm{Ph} 2$

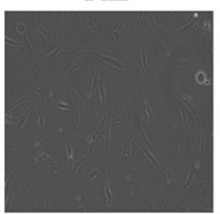

FS2

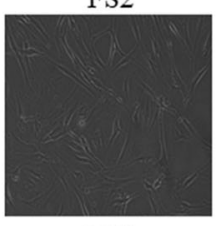

FS4

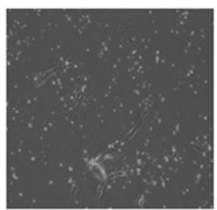

FS6

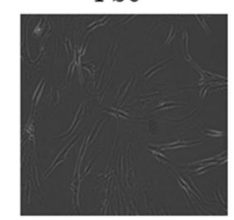

FS8

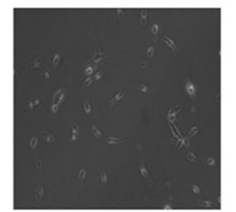

FS10

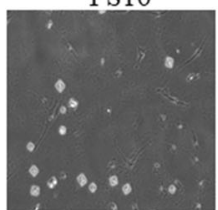

B

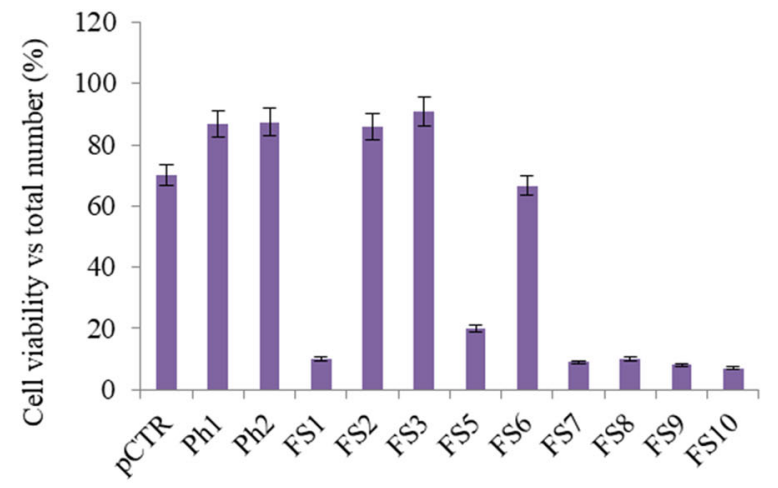

C

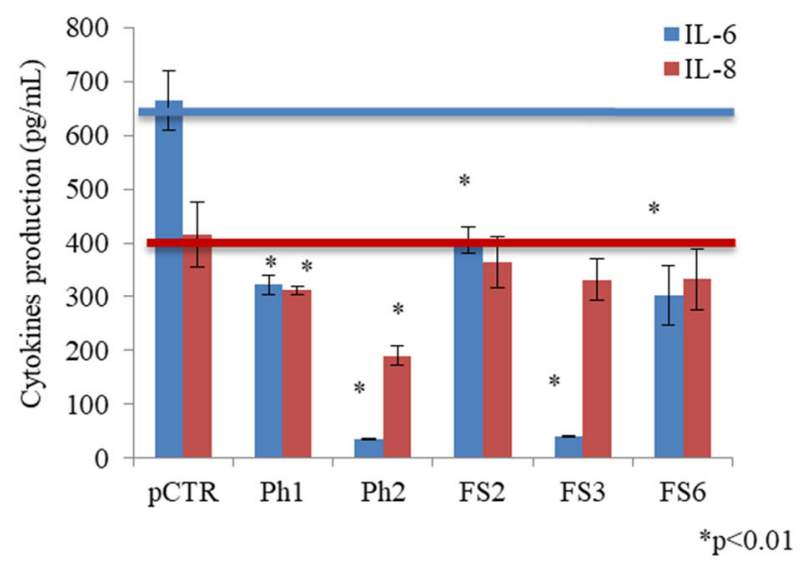

FS7

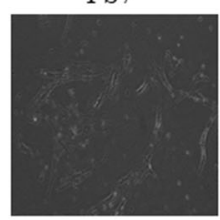

FS9

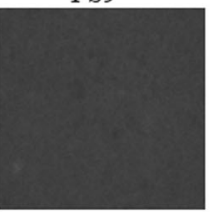

D
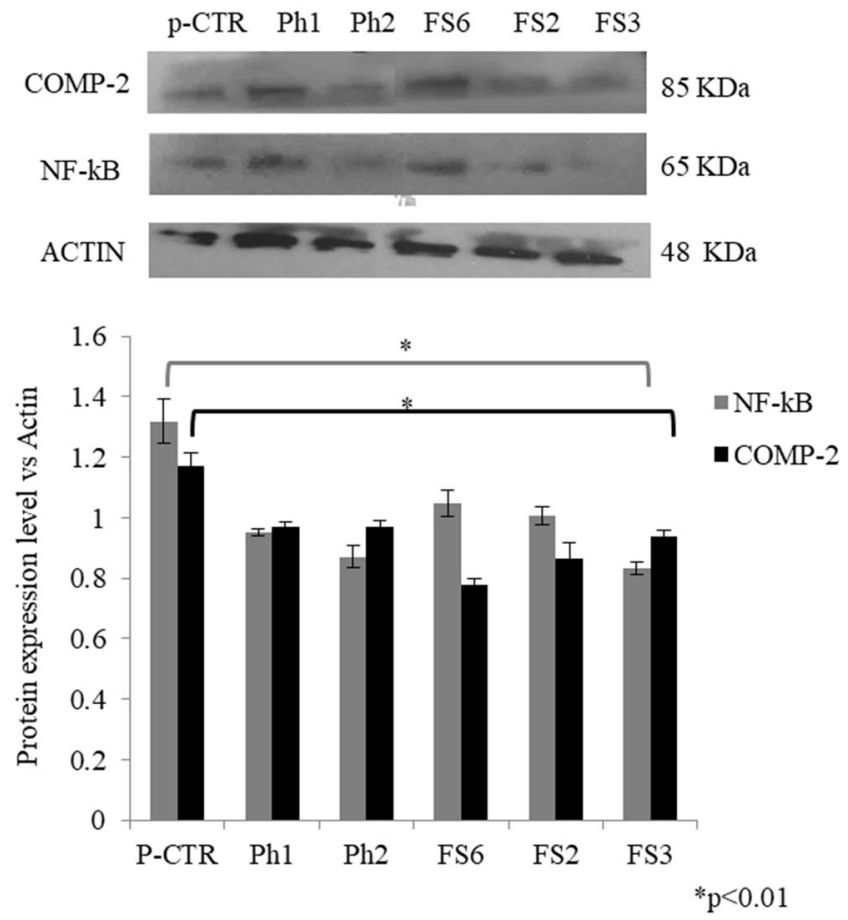
4Fig. 5 Biological activity on synoviocytes. a Cell picture panel in the presence of food supplements and pharmaceutical grade products versus pathological CTR. b Cell viability using trypan blue staining. c IL-6 and IL-8 cytokine quantification through ELISA. d Western blotting analyses of $\mathrm{NF}-\kappa \mathrm{B}$ and COMP-2 versus actin housekeeping protein normalization. $T$ test analyses $\left({ }^{*} p<0.01\right)$ were performed to compare the significance of both pharmaceutical grade CS samples and food supplements on IL- 6 and IL-8 production, on NF- $\mathrm{KB}$ and COMP-2 protein expression by synoviocytes with respect to pathological non-treated cells

treatment, $\mathrm{Ph} 2$ was more effective than $\mathrm{Ph} 1$ with a slightly better reduction in NF- $\mathrm{B}$ expression (Fig. 5d). Also the food supplements decreased NF- $\kappa \mathrm{B}$ protein levels compared to pCTR, in particular FS3 with a reduction by about 1.2-fold. Instead, COMP-2 levels were reduced with more efficacy by Ph2, FS2, and FS3; respectively by about 1.3-, 1.5-, and 1.6-fold.

Statistical Analysis The data are presented as the mean \pm SD. When there was no variability in the values for a group, a one-sample $t$ test was used to compare the group mean to that value; otherwise a two-sample $t$ test was used to compare group means. $p<0.01$ was considered significant in each of the comparative analyses.

\section{DISCUSSION}

The biological activity of CS and GlcN food supplements could greatly vary according to their quantity and purity $[13,14]$, the structural heterogeneity of the CS (e.g., different molecular weight, mixed origin, bound proteins), and moreover the presence of other components, contaminants, or adulterants $[22,23]$. To fill the gap caused by the absence of any specific official indication, in this study a systematic approach was designed to analyze at the chemical, biochemical, and biological level 10 European FS samples, in comparison with two pharmaceutical grade products. Compared to $\mathrm{Ph}$ grade CS-based products, most of the FS samples proved to have high insoluble portions, probably due to the presence of plant extract residues. Results showed that 7/10 FS presented insolubles higher than $25 \%$ of the total dry weight. The analyses of FS chromatographic profiles showed the presence of many species in the samples, especially unretained molecules, and highlighted a very low representativeness of CS main peaks compared to pharmaceutical grade samples. This may be ascribed to less standardized and less robust (validated) manufacturing processes. A recently developed method exploiting high-performance anionexchange chromatography was used to determine both the CS and GlcN contents and to quantify the KS contamination. In the majority of the analyzed European food supplements, the CS and GlcN contents were lower than the declared contents (in $9 / 10$ and $7 / 10$, respectively), with over half of them presenting differences higher than $10 \%[24,27]$. Following the analytical characterization, the samples of both $\mathrm{Ph}$ and FS were compared for their biological activity by using three human primary cell models: (a) the first based on chondrocytes isolated from nasal cartilage and exposed to IL$1 \beta$ to mimic osteoarthritis in vitro conditions $[30,31]$; (b) the second using articular chondrocytes; and (c) the third based on synoviocytes isolated from knee cartilage and synovial fluid of OA-affected patients, respectively [32]. The human primary cell models were already used in our laboratory for previous experiments aimed at evaluating the biological activity of different sourced CS [30, 33].

Synovial cell cultures exposed to lipopolysaccharide treatment, instead, were reported in the literature as a model to study the protective and anti-inflammatory effects of $\mathrm{HA}$ alone or in combination with $\mathrm{CS}$ and GlcN $[34,35]$. In addition, through the NF- $\mathrm{kB}$ activation and IL-8 production, the synovial cells have been used to evaluate the anti-inflammatory response of food supplements with other potentially bioactive molecules (e.g., HA, collagen peptides, Boswellia serrata, devil's claw, or curcumin). Specifically, $\mathrm{NF}-\mathrm{\kappa B}$ is a signaling molecule known to play an important role in the regulation of inflammatory mediators involved in the pathogenesis of OA [36]. We therefore assessed this biomarker in the pathological primary cells, after treatment with $\mathrm{Ph}$ and/or FS products, finding a significant 
decrease in Ph1- and Ph2-treated cells, while only one of the FS showed a similar positive behavior. Also, COMP-2 is strictly related to OA as a diagnostic and prognostic indicator of the disease severity and the effect of treatment [33]. The data from this experimental research clearly indicated that the lower control on the FS composition does not support their biological efficacy. Specifically, among 10 FS tested only three do not negatively affect cell viability and showed efficacy on the reduction of pro-inflammatory cytokines (e.g., IL6 and IL-8) and on the expression of NF- $\mathrm{KB}$ and COMP-2. On the other hand, the pharmaceutical grade products, $\mathrm{Ph} 1$ and $\mathrm{Ph} 2$, proved to have a very good biological activity. In particular, they sustain cell viability in pathological models and are able to reduce IL- 6 and IL- 8 production and also to modulate NF- $\mathrm{BB}$ and COMP-2 expression, proving that they have anti-inflammatory properties. Among the food supplements tested only FS2 and FS3 proved to be not cytotoxic for all the three cell-based models, while FS5 and FS6 reduced viability of nasal and articular chondrocytes. FS3 proved to be the best among the FS in terms of quality and bioactivity. In fact, it reduced IL-6 expression in all three cellular models investigated, but decreased IL-8 amounts only in nasal chondrocytes and synoviocytes. In vitro comparative analyses may improve knowledge on the mechanism of action, and also suggest that the diverse in vivo outcome, often reported in the scientific literature, may probably be related to the different product quality administered to patients [37]. In fact, compared to the pharmaceutical grade products, FS contained low quality CS, highly contaminated by keratan sulfate, and CS of multiple origins that was often present $[23,27]$.

Apart from data reported in the literature, all these additional analytical data perfectly match with the biological assay outcomes. Pharmaceutical grade products proved to be biologically active in all the proposed cellular models, while only three of the 10 screened food supplements presented appropriate biological activity. Specifically $\mathrm{Ph}$ products significantly reduced the secreted inflammatory cytokines, through the NF- $\mathrm{BB}$ pathway also significantly reducing COMP-2, considered a biomarker for negative prognosis on $\mathrm{OA}$ progression. These results support the ESCEO recommendation to use, among all chondroitin products available, the pharmaceutical grade prescription chondroitin products, for which the evidence base is unequivocal [38].

\section{Limitations of the Current Study}

The main limitation is due to the evaluation of bioactivity in in vitro models, which resemble the in vivo pathology but are not completely equivalent to a whole organism (animal, humans). In fact, in vitro tests are only one part of the global picture and pharmacokinetics-pharmacodynamics studies as well as the demonstration of a clinical equivalence/noninferiority would also be of great interest [38]. In addition, the FS studies were from European countries so corresponding American and Asian products should be analyzed for a global view of the market situation, and the benefit/risk ratio for patients.

\section{CONCLUSIONS}

In this paper a systematic assessment of $10 \mathrm{CS}$ and GlcN-based food supplements commercialized throughout European countries was performed and compared to pharmaceutical CS. The results indicated that most of them do not perfectly match the descriptions reported on the labels in that they present lower CS and GlcN contents. On the other hand, Ph-based products were consistent in purity and titer. Addition of Ph samples elicited beneficial effects at multiple levels in the three human primary cell models tested in vitro. In contrast, a minority of FS assayed were able to sustain cell viability and to reduce inflammation. These results may help researchers and clinicians to improve knowledge and understanding when analyzing and interpreting clinical outcomes in scientific reports. In general, on the basis of these data, only a small percentage of FS products containing CS might have similar biological properties to pharmaceutical grade CS. Most FS containing CS are of uncertain quality with equivocal efficacy and doubtful safety in the treatment of OA. 


\section{ACKNOWLEDGEMENTS}

Funding. This work was partially supported by PON01_1226 NUTRAFAST, MIUR Ministero dell'Università e della Ricerca Scientifica. Bioteknet financed two short-term grants for graduate technicians. The journal's Rapid Service and Open Access fees were funded by IBSA $\mathrm{CH}$.

Authorship. All named authors meet the International Committee of Medical Journal Editors (ICMJE) criteria for authorship for this article, take responsibility for the integrity of the work as a whole, and have given their approval for this version to be published.

Disclosures. Antonietta Stellavato, Odile Francesca Restaino, Valentina Vassallo, Rosario Finamore, Carlo Ruosi, Elisabetta Cassese, Mario De Rosa, and Chiara Schiraldi have nothing to disclose.

Compliance with Ethics Guidelines. This article is not based on in vivo studies, on either animals or humans. However, the cells used were obtained by digestion of joint/cartilage tissues harvested from surgical procedures on human participants. The pieces would have been wasted and, as part of a collaboration scheme, these were used instead to set up in vitro models used in this experimental research project. The protocol was approved by the ethical committee of the University (AOUSUN) (protocol registration No. 0003711/2015 of the 16/05/2015; study title "Glicosamminoglicani e/o derivati biotecnologici utili alla rigenerazione tessutale"). Informed consent was obtained from all participants involved in this study.

Data Availability. All relevant data are reported within the manuscript. All relevant data are presented in the results section and only those are discussed in the discussion section.

Open Access. This article is distributed under the terms of the Creative Commons
Attribution-NonCommercial 4.0 International License (http://creativecommons.org/licenses/ by-nc/4.0/), which permits any noncommercial use, distribution, and reproduction in any medium, provided you give appropriate credit to the original author(s) and the source, provide a link to the Creative Commons license, and indicate if changes were made.

\section{REFERENCES}

1. Le Graverand-Gastineau MPH. OA clinical trials: current targets and trials for OA choosing molecular targets: what have we learned and where we are headed. Osteoarthr Cartil. 2009;17(11):1393-401.

2. Wang T, He C. Pro-inflammatory cytokines: the link between obesity and osteoarthritis. Cytokine Growth Factor Rev. 2018;44:38-50.

3. Conaghan PG, Kloppenburg M, Schett G, Bijlsma JW. Osteoarthritis research priorities: a report from EULAR ad hoc expert committee. Ann Rheum Dis. 2014;73:1442-5.

4. Chevalier X, Conrozier T. Access to highly purified chondroitin sulfate for appropriate treatment of osteoarthritis: a review. MA@PoC.2017;1(1):134-44.

5. Yun Z, Peng HZ, Wang W, et al. A20 inhibits the release of inflammatory cytokines by suppressing the activation of the nuclear factor-kappa B pathway in osteoarthritic fibroblast-like synoviocytes. Biochem Biophys Res Commun. 2019;508(3):877-81.

6. Hong R, Sur B, Yeom M, et al. Anti-inflammatory and anti-arthritic effects of the ethanolic extract of Aralia continentalis Kitag. In IL-1 $\beta$-stimulated human fibroblast-like synoviocytes and rodent models of polyarthritis and nociception. Phytomedicine. 2018;38:45-56.

7. Goldring MB, Otero M. Inflammation in osteoarthritis. Curr Opin Rheumatol. 2011;23(5):471-8.

8. Majeed MH, Sherazi SAA, Bacon D, Bajwa ZH. Pharmacological treatment of pain in osteoarthritis: a descriptive review. Curr Rheumatol Rep. 2018;20(12):88.

9. Agiba AM. Nutraceutical formulations containing glucosamine and chondroitin sulphate in the treatment of osteoarthritis: emphasis on clinical efficacy and formulation challenges. Int J Curr Pharm Res. 2017;2:1-7. 
10. Hochberg M, Chevalier X, Henrotin Y, Hunter DJ, Uebelhart D. Symptom and structure modification in osteoarthritis with pharmaceutical-grade chondroitin sulfate: what's the evidence. Curr Med Res Opin. 2013;29(3):259-67.

11. Davies PS, Graham SM, MacFarlane RJ, Leonidou A, Mantalaris A, Tsiridis E. Disease-modifying osteoarthritis drugs: in vitro and in vivo data on the development of DMOADs under investigation. Expert Opin Investig Drugs. 2013;22(4):423-41.

12. Jordan KM, Arden NK, Doherty M, et al. EULAR Recommendations 2003: an evidence based approach to the management of knee osteoarthritis: report of a Task Force of the Standing Committee for International Clinical Studies Including Therapeutic Trials (ESCISIT). Ann Rheum Dis. 2003;62(12):1145-55.

13. Zhang W, Doherty M, Arden N, et al. EULAR evidence based recommendations for the management of hip osteoarthritis: report of a task force of the EULAR Standing Committee for the International Clinical Studies Including Therapeutics (ESCISIT). Ann Rheum Dis. 2005;64:669-81.

14. Michel BA, Stucki G, Frey D, et al. Chondroitins 4 and 6 sulfate in osteoarthritis of the knee: a randomized, controlled trial. Arthritis Rheum. 2005;52:779-86.

15. Volpi N, Maccari F. Two analytical approaches to the evaluation of chondroitin sulfate in European food supplements. Sep Sci. 2009;1(1):23-8.

16. Volpi N, Maccari F. Quantitative and qualitative evaluation of chondroitin sulfate in dietary supplements. Food Anal Methods. 2008;1(3):195-204.

17. Shi YG, Meng YC, Li JR, Chen J, Liu YH, Bai X. Chondroitin sulfate: extraction, purification, microbial and chemical synthesis. Chem Tech Biotechnol. 2014;89(10):1445-65.

18. Collin EC, Carroll O, Kilcoyne M, Peroglio M, See W, Hendig D, Alini M, Grad S, Pandit A. Ageing affects chondroitin sulfates and their synthetic enzymes in the intervertebral disc. Signal Trans Target Ther (2017);2:17049.

19. Bruyere O, Cooper C, Pelletier JP, et al. An algorithm recommendation for the management of knee osteoarthritis in Europe and internationally: a report from a task force of the European Society for Clinical and Economic Aspects of Osteoporosis and Osteoarthritis (ESCEO). Semin Arthritis Rheum. 2014;44:253-63.

20. Bruyère $\mathrm{O}$, Cooper $\mathrm{C}$, Cutolo $\mathrm{M}$, Reginster JY. International endorsement of the ESCEO algorithm for management of knee osteoarthritis in clinical practice. Semin Arthritis Rheum. 2017;47(2):e10.

21. Bruyère $\mathrm{O}$, Honvo $\mathrm{G}$, Veronese $\mathrm{N}$, et al. An updated algorithm recommendation for the management of knee osteoarthritis from the European Society for Clinical and Economic Aspects of Osteoporosis, Osteoarthritis and Musculoskeletal Diseases (ESCEO). Semin Arthritis Rheum. 2019. https://doi. org/10.1016/j.semarthrit.2019.04.008.

22. Restaino OF, De Rosa M, Cimini D, Schiraldi C. Manufacturing chondroitin sulfate: from animal source extraction to biotechnological production. In: Pomin VH, editor. Chondroitin sulfate: structure, uses and health implications. New York: Nova Science; 2013. p. 41-56.

23. Restaino OF, Finamore R, Diana P, et al. A multianalytical approach to better assess the keratan sulfate contamination in animal origin chondroitin sulfate. Anal Chim Acta. 2017;15(958):59-70.

24. Funderburgh JL. Keratan sulfate: structure, biosynthesis, and function. Glycobiology. 2000;10(10): 951-8.

25. Pomin VH, Piquet AA, Pereira MS, Mourão PAS. Residual keratan sulfate in chondroitin sulfate formulations for oral administration. Carbohydr Polym. 2012;90:839-46.

26. Bottelli S, Grillo G, Barindelli E, Nencioni A, Di Maria A, Fossati T. Validated high-performance anion-exchange chromatography with pulsed amperometric detection method for the determination of residual keratan sulfate and other glucosamine impurities in sodium chondroitin sulfate. J Chromatogr A. 2017;7(1505):43-9.

27. Restaino OF, Finamore R, Stellavato A, et al. European chondroitin sulfate and glucosamine food supplements: a systematic quality and quantity assessment compared to pharmaceuticals. Carbohydr Polym. 2019;222:114984.

28. Honvo $\mathrm{G}$, Bruyère $\mathrm{O}$, Geerinck $\mathrm{A}$, Veronese $\mathrm{N}$, Reginster JY. Efficacy of chondroitin sulfate in patients with knee osteoarthritis: a comprehensive meta-analysis exploring inconsistencies in randomized, placebo-controlled trials. Adv Ther. 2019;36(5):1085-99.

29. Bradford MM. A rapid and sensitive method for the quantitation of microgram quantities of protein utilizing the principle of protein-dye binding. Anal Biochem. 1976;72:248-54.

30. Stellavato A, Tirino V, De Novellis F, et al. Biotechnological chondroitin a novel glycosaminoglycan with remarkable biological 
function on human primary chondrocytes. J Cell Biochem. 2016;117(9):2158-69.

31. Calamia V, Ruiz-Romero C, Rocha B, FernandezPuente P, Mateos J. Pharmacoproteomic study of the effects of chondroitin and glucosamine sulfate on human articular chondrocytes. Arthritis Res Ther. 2010;12(4):R138.

32. Stellavato A, Vassallo V, La Gatta A, et al. Novel hybrid gels made of high and low molecular weight hyaluronic acid induce proliferation and reduce inflammation in an osteoarthritis in vitro model based on human synoviocytes and chondrocytes. Biomed Res Int. 2019;23:4328219.

33. Stellavato A, de Novellis F, Reale S, De Rosa M, Schiraldi C. Hybrid complexes of high and low molecular weight: evaluation using an in vitro model of osteoarthritis. J Biol Regul Homeost Agents. 2016;30:7-16.

34. Kilborne AH, Hussein H, Bertone AL. Effects of hyaluronan alone or in combination with chondroitin sulfate and N-acetyl-d-glucosamine on lipopolysaccharide challenge-exposed equine fibroblast-like synovial cells. Am J Vet Res. 2017;78(5):579-88.

35. Yamagishi Y, Someya A, Imai K, Nagao J, Nagaoka I. Evaluation of the anti inflammatory actions of various functional food materials including glucosamine on synovial cells. Mol Med Rep. 2017;16(2):1353-9.

36. Qian YQ, Feng ZH, Li XB, et al. Downregulating PI3 K/Akt/NF- $\mathrm{BB}$ signaling with allicin for ameliorating the progression of osteoarthritis: in vitro and vivo studies. Food Funct. 2018;9(9):4865-75.

37. Hochberg MC, Berry S, Broglio K, et al. Mixed treatment comparison of efficacy and tolerability of biologic agents in patients with rheumatoid arthritis. Curr Med Res Opin. 2013;29(10):1213-22.

38. Bruyère $\mathrm{O}$, Cooper $\mathrm{C}$, Al-Daghri NM, Dennison EM, Rizzoli R, Reginster JY. Inappropriate claims from non-equivalent medications in osteoarthritis: a position paper endorsed by the European Society for Clinical and Economic Aspects of Osteoporosis, Osteoarthritis and Musculoskeletal Diseases (ESCEO). Aging Clin Exp Res. 2018;30(2):111-7. 